\title{
Years of Life Lost due to Suicide in Southern Iran 2011-18: A Population-Based Study
}

\author{
Alireza Mirahmadizadeh, MD, PhD; Fariba Rezaei, MS²; Leila Moftakhar, MS³ Neda Heiran, BA4 Habibollah Azarbakhsh, MS ${ }^{*}$
}

${ }^{1}$ Non-Communicable Diseases Research Center, Shiraz University of Medical Sciences, Shiraz, Iran

${ }^{2}$ Shiraz University of Medical Sciences, Mental Health, Shiraz University of Medical Sciences, Shiraz, Iran

${ }^{3}$ Student Research Committee, Shiraz University of Medical Sciences, Shiraz, Iran

${ }^{4}$ School of Nursing and Midwifery, Zahedan University of Medical Sciences, Zahedan, Iran

\begin{abstract}
Background: Suicide is a major public health concern with diversity in epidemiological aspects and applied methods. In this study, we estimate years of life lost (YLLs) related to completed suicidal in the Fars province, southern Iran.

Methods: Our study included data of all mortality events during 2011-2018 from Fars Suicide Surveillance System (FSSS). The validity of qualitative and quantitative variables was assessed through contrasting data between different sources and phone call justification. Case-fatality rates, age-specific and gender-specific mortality rates, ASR (age standardized rate), and YLLs through WHO's 2015 "YLL template" were calculated.

Results: During the study period, 2384 mortalities with a mean age of $32.73 \pm 15.65$ had been registered. Most of them were males (male: $70.05 \%$ vs. female: $29.95 \%$; male-female ratio: 2.33 ), hanging was the most frequent method (29.94\%), and an increasing pattern in successful suicidal attempts was observed. The total YLLs were calculated to be 58669 years (14.71 per 1000 persons). Regardless of year or gender, suicide had the largest YLLs amongst those aged 15-29 years.

Conclusion: Regarding the increasing trend in YLLs and observing the highest rate of successful suicidal attempts amongst active and productive members of community, a comprehensive inter organizational reaction is demanded.

Keywords: Burden, Premature death, Suicide, YLL

Cite this article as: Mirahmadizadeh A, Rezaei F, Moftakhar L, Heiran N, Azarbakhsh H. Years of life lost due to suicide in southern iran 2011-18: a population-based study. Arch Iran Med. 2022;25(1):12-16. doi: 10.34172/aim.2022.03
\end{abstract}

Received: December 29, 2020, Accepted: April 13, 2021, ePublished: January 1, 2022

\section{Introduction}

Suicide is defined as a deliberate attempt of self-killing. ${ }^{1}$ It is a major public health concern that carries a substantial burden in all communities, accounting for 1.4 and $1.48 \%$ of all mortalities in 2012 and 2015, respectively. ${ }^{2}$ Globally, it has been estimated that 800000 suicide events occur annually, $78 \%$ of which pertain to low- and middleincome countries. ${ }^{3}$ The World Health Organization (WHO) reported that suicide is one of the three leading causes of mortality among the 15-44 years age group, ${ }^{4}$ since adolescents and early adulthood claim the highest share of premature death due to suicidal attempts. ${ }^{5}$ Also, in Asia, suicide is one of the main fifteen causes of mortality. Available data suggest that for each successful suicidal attempt, 8 to 40 attempts occur. ${ }^{6}$

Suicide is a complex and multifactorial phenomenon, which is linked to individual, familial, and social factors, ${ }^{7}$ including male gender (But suicidal attempts are more common in women), younger age, mental disorders like depression or schizophrenia, white race, low income, right to keep and bear arms, job loss, drug abuse, etc., ${ }^{8,9}$ A diversity exists in the most frequent methods of suicide in different regions; however, hanging, drowning, falling, poisoning, and firearms are more prevalent. $^{5}$

In Iran, the rate of annual suicidal attempts has been reported at 3.6 per 100000 people, which is lower than worldwide estimates. ${ }^{1}$ Furthermore, while the premature death related to suicidal attempts in Iran is substantially lower than European countries, the measure of years of life lost (YLLs) in Iran is higher than European countries at younger ages. ${ }^{3}$ The mortality and morbidity of suicidal attempts in Iran cause YLLs of 200 in every 100000 individuals, which ranks as the fifth leading cause of premature death. ${ }^{10}$

The Fars province with 122608 square kilometers is located in southern Iran and is the fourth most populous province in the country with 4851274 residents in $2016 .{ }^{11}$ In this study, we sought to estimate mortality rate, agespecific and gender-specific mortality rate, and YLLs related to completed suicide in the Fars province, during an 8-year period from 2011 to 2018.

\section{Materials and Methods \\ Settings and Data Acquisition}

In this cross-sectional study, we included all mortality events in the Fars province during 2011-2018. This data was acquired from Mental Health and Suicide Surveillance Systems that is Fars Suicide Surveillance System (FSSS). Conventionally, in FSSS, data collection and data analysis for research purposes are permitted by patients or their 
parents/relatives/caregivers through written informed consents. These data would be codified, except for their identity information.

Worth noting, relatives were not directly contacted other than a randomly selected $5 \%$ to confirm the validity of surveillance data by contrasting with verbally declared autopsy results.

We investigated the data of all affiliated centers, mortality and morbidity of all diseases as well as their causes, including suicidal attempts. FSSS registers demographic information, medical history, suicidal history, as well as causes, methods and outcome of suicidal events. The surveillance system data were rechecked with data of psychiatric clinics, hospitals, local forensic medicine office, death registries, urban and rural health centers, medical toxicology centers, and emergency medicine departments. Then, duplicates were excluded, which resulted in 2384 events of successful suicide.

The validity of qualitative and quantitative variables was assessed through contrasting data between different sources and phone call justification

The population of the province was estimated using local health centers' databases and national census reports, with respect to the estimated annual population growth, which yielded a 4851274-person population in 2018.

\section{Statistical Analysis}

Descriptive analysis was carried out using the Statistical Package for Social Sciences (SPSS) (Version 22.0 for Windows, Released 2013. Armonk, NY: IBM Corp.), which included frequency (percent) of mortality events, sex ratio, and mean \pm Standard Deviation $(S D)$ of age at the time of suicidal attempt.

To calculate age-specific and gender-specific mortality rates, first, case-fatality rates were obtained; then based on the 2013 standards for low- and medium-income countries, ASR (age standardized rate) measures were obtained. ${ }^{12}$

YLLs analysis was carried out using the WHO's 2015 "YLL template", which ran in Microsoft Excel spreadsheet (2007). YLLs were calculated by two different methods. To do this, we applied the simple method (A) and the complex method (B) that are represented in the WHO's second edition of "National burden of disease studies: a practical guide" booklet in 2001(Table 1). ${ }^{13}$

YLLs were estimated in 5-year age intervals for each gender. Afterwards, those were integrated and reconstructed to 10-to-15-year age intervals, comprising

Table 1. Methods A and B in Calculating YLLs Related to Successful Suicidal Attempts During 2011-2018

\begin{tabular}{ll}
\hline Method A & SEYLL $=N^{*} L$ \\
\hline Method B $\begin{array}{l}\text { SEYLL }=N \text { Ce } \\
\text { a }[-(\beta) /(\beta+r) a-1]\end{array}$
\end{tabular}

$\mathrm{N}$, Number of mortalities in a specific age group and gender; $L$, Standardized QoL (quality of life) in a specific age group and gender; $r$, Discounting rate, $0.03 ; \beta$, Age weight, $0.04 ; \mathrm{C}$, Correction factor of age weight, $0.1658 ; \mathrm{a}$, Age at death; e, 2.71828 .
$5-14,15-29,30-44,45-59,60-69,70-79$, and $>80$ years of age.

Results

During 2011-2018 in the Fars province, 2384 mortalities with a mean age of $32.73 \pm 15.65$ had been confirmed due to suicide. Males were dominant (male: $70.05 \%$ vs. female: 29.95\%; male-female ratio: 2.33 ) and had a higher age at the time of suicidal attempt (male: $34.07 \pm 16.49$, female: $30.34 \pm 14.79)$. The main method used for suicide was hanging (29.94\%) (Table 2). Generally, the mortality rate due to suicide in the Fars province showed an increasing pattern during the study period, and the highest mortality rate was observed in the last year of the study (frequency: 400, 9.68 per 100000 ) (Table 3).

The total YLLs due to premature death in the 8-year period were 40659 years (20.16 per 1000 persons) in males, 18010 years (9.14 per 1000 persons) in females and 58669 years (14.71 per 1000 persons) in total. Regardless of year or gender, suicide had the largest YLLs in persons aged $15-29$ years, followed by $30-44,45-59$, and 5-14

Table 2. Absolute and Relative Frequency of Successful Suicidal Attempts in the Fars Province, Iran During 2011-2018

\begin{tabular}{|lcl|}
\hline Variable & Frequency & Percent \\
\hline Marital status & 1011 & \\
\hline Single & 898 & 42.40 \\
\hline Married & 475 & 37.66 \\
\hline Missing value & & 19.94 \\
\hline Suicide method & 714 & \\
\hline Hanging & 301 & 29.94 \\
\hline Drug overdose & 274 & 16.81 \\
\hline Toxic agent & 128 & 11.49 \\
\hline Firearms & 18 & 5.36 \\
\hline Cold weapon & 233 & 0.75 \\
\hline Burning & 2 & 9.77 \\
\hline Alcohol poisoning & 2 & 0.08 \\
\hline Drowning & 35 & 0.08 \\
\hline Falling & 46 & 1.46 \\
\hline Others & 531 & 1.92 \\
\hline Missing value & & 22.28 \\
\hline
\end{tabular}

Table 3. Frequency and Mortality Rate (Per 100000) of Successful Suicidal Attempt in the Fars Province, Iran During 2011-2018

\begin{tabular}{ccccccc}
\hline \multirow{2}{*}{ Year } & \multicolumn{2}{c}{ Male } & \multicolumn{2}{c}{ Female } & \multicolumn{2}{c}{ Total } \\
\cline { 2 - 7 } & Frequency & Rate & Frequency & Rate & Frequency & Rate \\
\hline 2011 & 193 & 9.52 & 73 & 3.81 & 266 & 6.90 \\
\hline 2012 & 143 & 7.29 & 78 & 4.03 & 221 & 5.67 \\
\hline 2013 & 208 & 10.48 & 113 & 5.79 & 321 & 8.16 \\
\hline 2014 & 222 & 11.07 & 97 & 4.93 & 319 & 8.03 \\
\hline 2015 & 248 & 12.23 & 90 & 4.53 & 338 & 8.42 \\
\hline 2016 & 242 & 11.80 & 80 & 3.99 & 322 & 7.94 \\
2017 & 128 & 6.17 & 68 & 3.36 & 196 & 4.79 \\
2018 & 286 & 13.65 & 114 & 5.59 & 400 & 9.68 \\
\hline
\end{tabular}


age groups (Tables 4 and 5). In addition, hanging (17515 years, 4.39 per 1,000 persons) claimed the largest YLLs among different methods used for suicide (Table 6).

\section{Discussion}

In the present study, we investigated YLLs related to successful suicidal attempts in the Fars province, Iran during 2011-2018. We found that the suicide mortality rate increased from 6.9 in 2011 to 9.6 in 100000 persons in 2018, and this upward trend was observed in both genders. We believed that this increase could be linked to economic crises that might be accompanied by psychological problems, substance abuse, unemployment, financial issues and other consequences.

Successful suicide and its related YLLs were higher in men, which is consistent with many other studies. ${ }^{2,8,14-16}$ This observation might be linked to several reasons. First, women often suicide to direct attention and do not apply life-threatening suicidal methods. Second, men are more prone to be in a high-risk social condition; for example, men consume psychedelic and addictive drugs much

Table 4. Age-specific and gender-specific YLLs* related to successful suicidal attempt in the Fars province, Iran during 2011-2018.

\begin{tabular}{|c|c|c|c|c|c|c|c|c|}
\hline 2018 & 2017 & 2016 & 2015 & 2014 & 2013 & 2012 & 2011 & Age-groups \\
\hline \multicolumn{9}{|l|}{ Male } \\
\hline 203 & 0 & 231 & 260 & 116 & 145 & 29 & 87 & $5-14$ \\
\hline 3431 & 1635 & 2698 & 3484 & 3166 & 2998 & 2473 & 2945 & $15-29$ \\
\hline 2154 & 921 & 2230 & 1586 & 1596 & 1263 & 592 & 1204 & $30-44$ \\
\hline 904 & 315 & 558 & 514 & 525 & 559 & 310 & 365 & $45-59$ \\
\hline 121 & 15 & 111 & 167 & 115 & 67 & 114 & 128 & $60-69$ \\
\hline 28 & 8 & 53 & 53 & 10 & 46 & 30 & 20 & $70-79$ \\
\hline 6 & 17 & 6 & 12 & 6 & 13 & 0 & 16 & +80 \\
\hline 6847 & 2911 & 5887 & 6076 & 5534 & 5091 & 3548 & 4765 & Total \\
\hline \multicolumn{9}{|c|}{ Female } \\
\hline 59 & 88 & 147 & 117 & 59 & 88 & 117 & 59 & $5-14$ \\
\hline 1497 & 980 & 1203 & 1276 & 1331 & 1917 & 1229 & 1362 & $15-29$ \\
\hline 684 & 452 & 550 & 654 & 663 & 580 & 505 & 329 & $30-44$ \\
\hline 202 & 126 & 124 & 210 & 333 & 247 & 118 & 120 & $45-59$ \\
\hline 159 & 49 & 42 & 30 & 49 & 63 & 16 & 16 & $60-69$ \\
\hline 27 & 0 & 12 & 18 & 9 & 21 & 32 & 0 & $70-79$ \\
\hline 23 & 0 & 0 & 0 & 7 & 0 & 0 & 11 & +80 \\
\hline 2651 & 1695 & 2078 & 2305 & 2451 & 2916 & 2017 & 1897 & Total \\
\hline \multicolumn{9}{|l|}{ Total } \\
\hline 262 & 88 & 378 & 377 & 175 & 233 & 146 & 146 & 5-14 \\
\hline 4928 & 2615 & 3901 & 4760 & 4497 & 4915 & 3702 & 4307 & $15-29$ \\
\hline 2838 & 1373 & 2780 & 2240 & 2259 & 1843 & 1097 & 1533 & $30-44$ \\
\hline 1106 & 441 & 682 & 724 & 858 & 806 & 428 & 485 & $45-59$ \\
\hline 280 & 64 & 153 & 197 & 164 & 130 & 130 & 144 & $60-69$ \\
\hline 55 & 8 & 65 & 71 & 19 & 67 & 62 & 20 & $70-79$ \\
\hline 29 & 17 & 6 & 12 & 13 & 13 & 0 & 27 & +80 \\
\hline 9498 & 4606 & 7965 & 8381 & 7985 & 8007 & 5565 & 6662 & Total \\
\hline
\end{tabular}

*YLL, years of life lost
Table 5. Age-Specific and Gender-Specific YLLs Per 1000 Person Related to Successful Suicidal Attempt in the Fars Province, Iran During 2011-2018

\begin{tabular}{|c|c|c|c|c|c|c|c|c|}
\hline Age Groups & 2011 & 2012 & 2013 & 2014 & 2015 & 2016 & 2017 & 2018 \\
\hline \multicolumn{9}{|l|}{ Male } \\
\hline $5-14$ & 0.28 & 0.09 & 0.45 & 0.36 & 0.79 & 0.69 & 0 & 0.59 \\
\hline $15-29$ & 4.28 & 3.72 & 4.68 & 5.14 & 5.89 & 4.75 & 3.01 & 6.61 \\
\hline $30-44$ & 2.48 & 1.16 & 2.36 & 2.86 & 2.72 & 3.68 & 1.46 & 3.29 \\
\hline $45-59$ & 1.26 & 1.03 & 1.80 & 1.63 & 1.55 & 1.63 & 0.89 & 2.50 \\
\hline $60-69$ & 1.59 & 1.29 & 0.70 & 1.11 & 1.50 & 0.93 & 0.11 & 0.90 \\
\hline 70-79 & 0.35 & 0.54 & 0.82 & 0.18 & 0.99 & 1.01 & 0.15 & 0.55 \\
\hline+80 & 0.48 & 0 & 0.38 & 0.17 & 0.35 & 0.17 & 0.48 & 0.17 \\
\hline Total & 2.45 & 1.80 & 2.56 & 2.75 & 2.99 & 2.87 & 1.40 & 3.26 \\
\hline ASR & 2.14 & 1.61 & 2.31 & 2.50 & 2.82 & 2.64 & 1.32 & 3.20 \\
\hline \multicolumn{9}{|l|}{ Female } \\
\hline $5-14$ & 0.20 & 0.39 & 0.29 & 0.19 & 0.37 & 0.46 & 0.27 & 0.18 \\
\hline $15-29$ & 2.00 & 1.88 & 3.06 & 2.38 & 2.23 & 2.20 & 1.89 & 3.04 \\
\hline $30-44$ & 0.68 & 1.00 & 1.10 & 1.20 & 1.14 & 0.92 & 0.72 & 1.06 \\
\hline $45-59$ & 0.41 & 0.39 & 0.80 & 1.06 & 0.64 & 0.37 & 0.36 & 0.57 \\
\hline $60-69$ & 0.17 & 0.16 & 0.59 & 0.43 & 0.25 & 0.33 & 0.36 & 1.12 \\
\hline $70-79$ & 0 & 0.60 & 0.39 & 0.16 & 0.33 & 0.22 & 0 & 0.50 \\
\hline+80 & 0.37 & 0 & 0 & 0.23 & 0 & 0 & 0 & 0.73 \\
\hline Total & 0.99 & 1.04 & 1.49 & 1.27 & 1.16 & 1.03 & 0.83 & 1.30 \\
\hline ASR & 0.84 & 0.92 & 1.35 & 1.18 & 1.08 & 1.01 & 0.83 & 1.33 \\
\hline \multicolumn{9}{|l|}{ Total } \\
\hline 5-14 & 0.24 & 0.23 & 0.37 & 0.27 & 0.58 & 0.58 & 0.13 & 0.39 \\
\hline $15-29$ & 3.14 & 2.81 & 3.88 & 3.82 & 4.09 & 3.50 & 2.46 & 4.88 \\
\hline $30-44$ & 1.58 & 1.08 & 1.73 & 2.04 & 1.94 & 2.31 & 1.09 & 2.19 \\
\hline $45-59$ & 0.84 & 0.71 & 1.31 & 1.35 & 1.10 & 1.01 & 0.63 & 1.55 \\
\hline $60-69$ & 0.84 & 0.70 & 0.64 & 0.76 & 0.85 & 0.62 & 0.24 & 1.01 \\
\hline $70-79$ & 0.18 & 0.57 & 0.61 & 0.17 & 0.66 & 0.61 & 0.07 & 0.52 \\
\hline+80 & 0.43 & 0 & 0.20 & 0.20 & 0.18 & 0.09 & 0.25 & 0.43 \\
\hline Total & 1.72 & 1.42 & 2.03 & 2.03 & 2.08 & 1.96 & 1.12 & 2.29 \\
\hline ASR & 1.49 & 1.26 & 1.83 & 1.86 & 1.96 & 1.83 & 1.08 & 2.28 \\
\hline
\end{tabular}

YLL, years of life lost; ASR, Age standardized rate

Table 6. YLLs by External Causes of Death (Methods) Due to Successful Suicidal Attempt in the Fars Province, Iran During 2011-2018

\begin{tabular}{lcccccc}
\hline \multirow{2}{*}{$\begin{array}{l}\text { External Causes } \\
\text { of Death }\end{array}$} & \multicolumn{3}{c}{ YLLs (years) } & \multicolumn{3}{c}{$\begin{array}{c}\text { YLLs Rate (Per 1000 } \\
\text { Persons) }\end{array}$} \\
\cline { 2 - 7 } & Male & Female & Total & Male & Female & Total \\
\hline Hanging & 14172 & 3343 & 17515 & 7.02 & 1.69 & 4.39 \\
\hline Drug overdose & 6394 & 3955 & 10349 & 3.16 & 1.99 & 2.58 \\
\hline Toxic agent & 4397 & 2337 & 6734 & 2.17 & 1.18 & 1.68 \\
\hline Alcohol poisoning & 57 & 0 & 57 & 0.02 & 0 & 0.01 \\
\hline Firearms & 2889 & 381 & 3270 & 1.43 & 0.19 & 0.82 \\
\hline Cold weapon & 303 & 103 & 406 & 0.15 & 0.05 & 0.10 \\
\hline Burning & 1778 & 4132 & 5910 & 0.88 & 2.09 & 1.48 \\
\hline Drowning & 27 & 22 & 49 & 0.38 & 0.18 & 0.28 \\
\hline Other (N=83) & 782 & 361 & 1143 & 4.88 & 1.71 & 3.31 \\
\hline Unknown & 9860 & 3376 & 13236 & 0.01 & 0.02 & 0.01 \\
\hline YLL, years of life lost & & & & & & \\
\hline
\end{tabular}


more than women. Third, men implement more intense methods for suicide that obviously increase the chance of premature death.

The highest mortality rate and YLLs due to suicide were observed in the age group 15-29 years. The results of a study by Izadi et al in Iran is in-line with this finding. ${ }^{3}$ In addition, in a study conducted in China, the highest mortality was reported for the age group of 20-24 years. ${ }^{4}$ This concerning finding imposes a heavy economic burden on families and the society. Given the fact that adolescents and young adults may be highly influenced by psychological and emotional stimulants and importantly physical and social reforms occur at these ages, any disability in psychosocial adjustment will increase the chance of attempting suicide. Also, this age group may commit fatal suicidal attempts under pressure of various factors and such as imitating their friends, substance abuse, family conflicts, economic problems, unemployment and financial poverty. These problems put people under the influence of psychological pressures. Eventually, because the pressure of these problems exceeds the resilience of people, it leads to suicide in these

The most frequently used method of suicide in our study was hanging, which claimed the highest YLLs. This finding is in-line with many other studies, ${ }^{2,5,14}$ and apparently might not be dependent on geographical diversity. For instance, in a study conducted in Canada, $46 \%$ of suicidal attempts occurred by hanging. ${ }^{16}$ The pervasiveness of hanging could be due to its accessibility. Additionally, it does not require special tools and brings a fast outcome; for example, consuming lethal doses of toxins or drugs cannot be straightforward; on the other hand, hanging is not as agonizing and dreadful as some methods such as self-immolation.

We showed that YLLs related to successful suicidal attempts increased from 1.7 in 2011 to 2.2 per 100000 persons in 2018 for both genders, which is consistent with the increasing number of suicidal attempts in the same region. As mentioned earlier, this upward trend might be linked to the deteriorating socioeconomic status and social welfare. The WHO declares that the suiciderelated mortality rate in Iran is lower than many countries; nevertheless, premature death cannot be neglected in public health's policy making. ${ }^{3}$ Life expectancy in Iran is around the mid-70s that is far from the ages of 15-29, where most lethal suicides occurred. These members of community are active and productive; in other words, mortality at these ages carries a heavy economic burden for the society, and potentially causes demographic changes. Hence, this is on health policymakers to identify and appropriately respond to the causes of suicidal attempts by implementing preventive strategies according to the cultural norms and economic conditions of the region.

In conclusion, we found an increasing trend in YLLs during the study period. Also, the highest rate of successful suicidal attempts was observed in adolescence and early adulthood. A comprehensive reaction to these figures demands the so-called interorganizational system and integrated efforts between the health, justice and other related ministries.

Authors' Contributions

$\mathrm{AbH}$ was responsible for the field working including data collection and management and analysis. ML wrote manuscript. RF collected data. MA edited the final version of the manuscript. MA and HN wrote the part of article.

\section{Conflict of Interest Disclosures}

The authors declare that they have no competing interests.

\section{Data Availability}

Data will not be shared because the university from which the information is collected does not

consent to provide the information.

\section{Ethics Statement}

This study was approved by research ethics committee of Fars University of Medical sciences (Ethics code: IR.SUMS.REC 1395. S950).

\section{References}

1. Veisani Y, Delpisheh A, Mohamadian F, Valizadeh R. Trends of suicide attempts and completed suicide in Ilam province of Iran; a demographic analysis study. Bull Emerg Trauma. 2018;6(3):245-8. doi: 10.29252/beat-0603010.

2. Orlewska K, Orlewska E. Burden of suicide in Poland in 2012: how could it be measured and how big is it? Eur J Health Econ. 2018;19(3):409-17. doi: 10.1007/s10198-017-0892-8.

3. Izadi N, Mirtorabi SD, Najafi F, Nazparvar B, Nazari Kangavari $\mathrm{H}$, Hashemi Nazari SS. Trend of years of life lost due to suicide in Iran (2006-2015). Int J Public Health. 2018;63(8):993-1000. doi: 10.1007/s00038-018-1151-1.

4. Sun L, Zhang J. Potential years of life lost due to suicide in China, 2006-2010. Public Health. 2015;129(5):555-60. doi: 10.1016/j.puhe.2015.02.012.

5. Bachmann S. Epidemiology of suicide and the psychiatric perspective. Int J Environ Res Public Health. 2018;15(7):1425. doi: 10.3390/ijerph15071425.

6. Mokhtari AM, Sahraian S, Hassanipour S, Baseri A, Mirahmadizadeh A. The epidemiology of suicide in the elderly population in Southern Iran, 2011-2016. Asian J Psychiatr. 2019;44:90-4. doi: 10.1016/j.ajp.2019.07.027.

7. Mokhtari AM, Gholamzadeh S, Salari A, Hassanipour S, Mirahmadizadeh A. Epidemiology of suicide in 10-19 years old in southern Iran, 2011-2016: a population-based study on 6720 cases. J Forensic Leg Med. 2019;66:129-33. doi: 10.1016/j.jflm.2019.06.018.

8. Ortiz-Prado E, Simbaña K, Gómez L, Henriquez-Trujillo AR, Cornejo-Leon F, Vasconez E, et al. The disease burden of suicide in Ecuador, a 15 years' geodemographic crosssectional study (2001-2015). BMC Psychiatry. 2017;17(1):342. doi: 10.1186/s12888-017-1502-0.

9. Möller-Leimkühler AM. The gender gap in suicide and premature death or: why are men so vulnerable? Eur Arch Psychiatry Clin Neurosci. 2003;253(1):1-8. doi: 10.1007/ s00406-003-0397-6.

10. Hajebi A, Ahmadzad-AsI M, Davoudi F, Ghayyomi R. Trend of suicide in Iran during 2009 to 2012: epidemiological evidences from national suicide registration. Iran J Psychiatry Behav Sci. 2016;10(4):e4398. doi: 10.17795/ijpbs-4398.

11. Mirahmadizadeh A, Rezaei F, Mokhtari AM, Gholamzadeh S, Baseri A. Epidemiology of suicide attempts and deaths: a population-based study in Fars, Iran (2011-16). J Public Health (Oxf). 2020;42(1):e1-e11. doi: 10.1093/pubmed/fdy218. 
12. Sankoh O, Sharrow D, Herbst K, Whiteson Kabudula C, Alam $\mathrm{N}$, Kant $\mathrm{S}$, et al. The INDEPTH standard population for lowand middle-income countries, 2013. Glob Health Action. 2014;7:23286. doi: 10.3402/gha.v7.23286.

13. World Health Organization (WHO). National Burden of Disease Studies: A Practical Guide. 2nd ed. Geneva: WHO; 2001.

14. Poorolajal J, Esmailnasab N, Ahmadzadeh J, Azizi Motlagh T. The burden of premature mortality in Hamadan province in 2006 and 2010 using standard expected years of potential life lost: a population-based study. Epidemiol Health. 2012;34:e2012005. doi: 10.4178/epih/e2012005.

15. van Spijker BA, van Straten A, Kerkhof AJ, Hoeymans N, Smit F. Disability weights for suicidal thoughts and non-fatal suicide attempts. J Affect Disord. 2011;134(1-3):341-7. doi: 10.1016/j.jad.2011.05.020.

16. Skinner R, McFaull S, Draca J, Frechette M, Kaur J, Pearson $C$, et al. Suicide and self-inflicted injury hospitalizations in Canada (1979 to 2014/15). Health Promot Chronic Dis Prev Can. 2016;36(11):243-51. doi: 10.24095/hpcdp.36.11.02. 